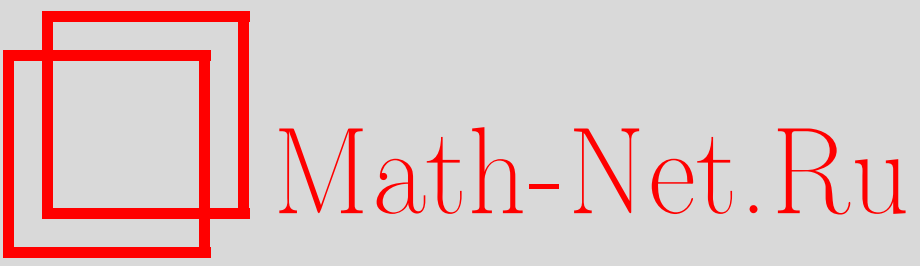

А. А. Вендина, О математическом моделировании процесса фрактальной миграции загрязнений в природных пористых системах, Вестн. Сам. гос. техн. ун-та. Сер. Физ.-мат. науки, 2011, выпуск 3(), 199-201

DOI: https://doi.org/10.14498/vsgtu1003

Использование Общероссийского математического портала Math-Net.Ru подразумевает, что вы прочитали и согласны с пользовательским соглашением

http://www.mathnet.ru/rus/agreement

Параметры загрузки:

IP : 54.164 .48 .24

26 апреля 2023 г., 17:25:25 
УДК 517.958:531.72

\section{О МАТЕМАТИЧЕСКОМ МОДЕЛИРОВАНИИ ПРОЦЕССА ФРАКТАЛЬНОЙ МИГРАЦИИ ЗАГРЯЗНЕНИЙ В ПРИРОДНЫХ ПОРИСТЫХ СИСТЕМАХ}

\section{А. А. Вендина}

Северо-Кавказский государственный технический университет, 355029, Ставрополь, просп. Кулакова, 2.

E-mail: Roven-ka@yandex.ru

Рассматриваются вопросъ математического моделирования нелинейных проиессов миграиии загрязнённых подземных вод, которые не могут быть описаны в рамках классического подхода в теории массопереноса. Для исследования пространственно-временных закономерностей нелинейных эббектов, обусловленнъх масштабной инвариантностъю, корректно поставлена нелокальная краевая задача для модельного дифберенциального уравнения нелинейной миграции.

Ключевые слова: миграция загрязнённых подземных вод, диффузия во фрактальных средах, дробные дибференииальные уравнения.

Процесс переноса загрязнений потоком подземных вод в природных пористых средах в рамках простых идеализированных представлений порового пространства и справедливости принципа локальности при определённой схематизации удовлетворительно описывается системой дифференциальных уравнений [1], состоящей из

- уравнения движения:

$$
v=-k \operatorname{grad} I, \quad u_{x}=-v_{x} c+D^{*} \frac{\partial c}{\partial x}, \quad u_{y}=-v_{y} c+D^{*} \frac{\partial c}{\partial y}
$$

- уравнения сохранения:

$$
\frac{\partial\left(p v_{x}\right)}{\partial x}+\frac{\partial\left(p v_{y}\right)}{\partial y}=\frac{\partial(m p)}{\partial t}, \quad m \frac{\partial c}{\partial t}=\frac{\partial u_{x}}{\partial x}+\frac{\partial u_{y}}{\partial y}-\frac{\partial N}{\partial t}
$$

- уравнения состояния:

$$
q=q\left(p ; p_{c} ; \mu\right), \quad f=f(\rho ; p), \quad \frac{\partial N}{\partial t}=\psi\left(N ; c ; \gamma_{i}\right)
$$

Здесь $v=v(x ; y ; t)$ - скорость движения потока грунтовых вод; $I=I(x ; y ; t)$ - напор; $c=c(x ; y ; t)$ - концентрация загрязняющих веществ в потоке грунтовых вод в точке $(x ; y)$ в момент времени $t ; m$ - активная пористость грунта; $D^{*}=D^{*}(x ; y ; t)-$ коэффициент конвективной диффузии; $p=p(x ; y ; t)$ - давление; $p_{c}$ - давление пласта; $\mu$ - коэффициент упругости; $\rho$ - плотность жидкости; $N=N(x ; y ; t)$ - концентрация вещества в твёрдой фазе; $\gamma_{i}$ - коэффициент скорости массообмена.

В силу уравнений (1)-(3), учитывающих совместное действие гравитационных и диффузионных сил, одномерный процесс загрязнения подземных вод описывается нелинейным дифференциальным уравнением в частных производных второго порядка параболического типа:

$$
m \frac{\partial c}{\partial t}=\frac{\partial}{\partial x}\left[D(x ; t) \frac{\partial c}{\partial x}\right]-\frac{\partial[v(x ; t) c]}{\partial x}-\frac{\partial N}{\partial t} .
$$

Алла Анатольевна Вендина, ст. преподаватель, каф. высшей математики. 
Обобщённое дифференциальное уравнение (4) в рамках эмпирических законов Фурье и Фика описывает процесс изменения во времени концентрации загрязнений в какой-либо точке среды вследствие разности переноса загрязнений фильтрационным потоком и гидродинамической дисперсии при наличии растворения и поступления в раствор солей и примесей, содержащихся в почвогрунтах. Построенные на основе обобщенного уравнения (4) решения различных начально-краевых задач $[1,2]$ не учитывают влияния неоднородной структуры порового пространства на процессы миграции и, как следствие, не отражают возникающих в них нелинейных эффектов, например, таких как «эффекты памяти» и конечность скорости распространения.

Современный метод математического моделирования возникающих в процессе миграции нелинейных явлений существенно использует идеи фрактальной геометрии в сочетании с формализмом дробного интегро-дифференцирования. Почва и почвогрунт хорошо интерпретируются как среды, структура пор которых образует множество с фрактальной размерностью $d_{f}$.

Пусть за промежуток времени $\Delta t$ происходит изменение концентрации загрязнений подземных вод на величину $\delta c=c_{t}^{\prime} \Delta t$. В соответствии с методами фрактального анализа [3] для скорости $c_{t}^{\prime}$ изменения концентрации загрязнения в процессе нелинейной миграции принимается гипотеза о том, что она определяется как дробная производная в смысле Римана-Лиувилля концентрации $c(x ; t)$ :

$$
c_{t}^{\prime} \approx D_{0 t}^{\alpha} c(x ; t)=\frac{1}{\Gamma(1-\alpha)} \frac{\partial}{\partial t} \int_{0}^{t} \frac{c(x ; \tau) d \tau}{(t-\tau)^{\alpha}},
$$

где $\alpha \in(0 ; 1)$ - порядок дробной производной. Для широких классов пористых сред полагают $\alpha \approx d_{f}$.

При выполнении гипотезы (5) обобщением уравнения (4) является нелокальное дифференциальное уравнение в частных производных второго порядка параболического типа с дробной производной по времени:

$$
m D_{0 t}^{\alpha} c=\frac{\partial}{\partial x}\left[D_{\alpha}(x ; t) \frac{\partial c}{\partial x}\right]-\frac{\partial\left[v_{\alpha}(x ; t) c\right]}{\partial x}-\frac{\partial N}{\partial t},
$$

где $D_{\alpha}-$ коэффициент фрактальной диффузии; $v_{\alpha}-$ фрактальная скорость.

Для однозначного описания нелинейной фрактальной миграции загрязнений к модельному уравнению миграции (6) присоединяют [4] видоизмененные начальные условия: условие типа Коши

$$
\lim _{t \rightarrow 0} D_{0 t}^{\alpha-1} c(x ; t)=f(x)
$$

или локальное весовое начальное условие

$$
\lim _{t \rightarrow 0} t^{1-\alpha} c(x ; t)=f(x)
$$

В условиях (7) дробный показатель $\alpha$, отражая фрактальную размерность пористой среды, соответствует доле пористых каналов или ветвей, открытых для протекания загрязнённых подземных вод в начальный момент времени. При решении задач с прикладной направленностью использование начальных условий типа Коши (7) оказывается непригодным для практических расчётов. Для преодоления указанной проблемы, как правило, дробная производная Римана-Лиувилля заменяется на нормализованную производную по Капуто и дифференциальное уравнение рассматривается с классическим начальным условием Коши

$$
c(x ; 0)=f(x) .
$$

Для исследования асимптотического режима миграции загрязнённых подземных вод в пористых средах в настоящей работе рассматривается следующая нелокальная начально-краевая задача. 
ЗАДАЧА.Найти в прямоуголъной области $\Omega=\{(x ; t): 0<x<l, 0<t<T\}$ регулярное решение $c=c(x ; t)$ уравнения $(6)$, удовлетворяющее начальному условию (8) и нелокальным краевым условиям

$$
\left.D_{\alpha} \frac{\partial c}{\partial x}\right|_{x=0}=D_{0 t}^{\alpha-1} c(0 ; t),\left.\quad \frac{\partial c}{\partial x}\right|_{x=l}=0
$$

Для поставленной задачи доказана теорема существования и единственности решения, предложен численно-разностный алгоритм решения, реализованный модифицированным методом прогонки. Анализ численной реализации расчётного алгоритма, выполненный для различных значений параметра $\alpha$, позволяет сделать вывод, что неустановившийся процесс загрязнения подземных вод с течением времени стремится к естественному равновесному состоянию, а процесс распределения концентрации загрязнений имеет затухающий асимптотический характер, что является характерным для нелинейного процесса переноса в пористых средах.

\section{БИБЛИОГРАФИЧЕСКИЙ СПИСОК}

1. Веригин Н. Н. О кинетике растворения и выноса солей при фильтрации воды в грунтах / В сб.: Растворение и выщелачивание горных пород. М.: Госстройиздат, 1957. С. 1727. [On Kinetics of the Dissolution of Salts During the Filtration of Water into Soils / In: Dissolution and Leaching of Rocks: Moscow, 1957. Pp. 17-27].

2. Вендина A. A. Математическое моделирование массопереноса в пористых средах // Научная жизнь, 2008. № 3. C. 21-24. [Vendina A. A. Mathematical modeling of mass transfer in porous mediums // Nauchnaya zhizn', 2008. no. 3. Pp. 21-24].

3. Нигматуллин Р. Р. Дробный интеграл и его физическая интерпретация // TMФ, 1992. T. 90, № 3. С. 354-368; англ. пер.: Nigmatullin R. R. Fractional integral and its physical interpretation // Theoret. and Math. Phys., 1992. Vol.90, no. 3. Pp. 242-251.

4. Нахушев А. М. Элементы дробного исчисления и их применение. Нальчик: КБНЦ РАН, 2000. 299 c. [Nakhushev A. M. Elements of Fractional Calculation and their Application. Nal'chik: KBNC RAN, 2003. 299 pp.]

Поступила в редакцию 03/III/2011;

в окончательном варианте - 07/VI/2011.

MSC: 26A33; 28A80

\section{ON THE MATHEMATICAL MODELING OF THE CONTAMINATED GROUNDWATER FRACTAL MIGRATION IN NATURAL POROUS SYSTEMS}

\section{A. A. Vendina}

North Caucasus State Technical University,

2, prosp. Kulakova, Stavropol', 355029, Russia.

E-mail: Roven-ka@yandex.ru

The problems of the mathematical modeling of the contaminated groundwater migration, which cannot be described in the context of the mass transfer theory classical approach, are considered. For the study of space-time conformity of nonlinear effects, determined by scaled invariance, the well-set nonlocal boundary problem for the model differential equation of nonlinear migration is proposed.

Key words: migration of contaminated groundwater, diffusion on fractals, fractional differential equations.

Original article submitted 03/III/2011; revision submitted $07 / \mathrm{VI} / 2011$.

Alla A. Vendina, Senior Teacher, Dept. of Higher Mathematics. 